E3S Web of Conferences 1, 09004 (2013)

DOI: $10.1051 / \mathrm{e} 3$ sconf/20130109004

(c) Owned by the authors, published by EDP Sciences, 2013

\title{
Trace metal contaminants in sediments and soils: comparison between ICP and XRF quantitative determination
}

\author{
A. Congiu ${ }^{1}$, S. Perucchini ${ }^{2}$ and P. Cesti $^{3}$ \\ 1 eni S.p.A. Direzione Ricerca e Innovazione Tecnologica, Centro Ricerche per le Energie non Convenzionali, Istituto \\ Eni Donegani, Via Fauser 428100 Novara, Italia, annalisa.congiu@eni.com \\ 2 eni S.p.A. Direzione Ricerca e Innovazione Tecnologica, Centro Ricerche per le Energie non Convenzionali, Istituto \\ Eni Donegani, Via Fauser 428100 Novara, Italia, sara.perucchini@eni.com \\ ${ }^{3}$ eni S.p.A. Direzione Ricerca e Innovazione Tecnologica, Centro Ricerche per le Energie non Convenzionali, Istituto \\ Eni Donegani, Via Fauser 428100 Novara, Italia, pietro.cesti@eni.com
}

\begin{abstract}
A mineralization method $\mathrm{HCl}$-free for heavy metals analysis in sediments and soils by DRC-ICP-MS was developed. The procedure, which uses concentrated nitric, hydrofluoric acid and hydrogen peroxide, was applied for the analysis of arsenic, cadmium, chromium, nickel and vanadium. The same samples were then analyzed, as pressed pellets, by wavelength dispersive X ray fluorescence (WD-XRF) using the dedicated PANalytical Pro Trace solution for the determination of trace elements. Comparison of ICP and XRF data showed good agreement for the elements under investigation, unless for chromium in soils, which recovery was not complete.
\end{abstract}

Key words: heavy metals, environmental samples, DRC-ICP-MS, WD-XRF, trace analysis

\section{Introduction}

The accurate determination of heavy metals content in environmental samples is of primary importance and it still represents a significant challenge. Taking in account the complexity of the matrices involved, the identification of reliable and fast methods for this application is a difficult goal to achieve.

ICP is a technique widely used for these purposes. Despite the introduction of close vessel microwave assisted mineralization system has been a crucial improvement, the sample pretreatment step is always a source of heavy uncertainty. In literature the problem of efficiency of the analyte recovery by varying the leaching/extraction procedure is widely debated over many years (Baffi et al. 2002, Hassan Nouri M. et al., 2007; Quevauviller et al. 1996, Sandroni and Smith, 2002; Wang et al. 2004).

Wavelength dispersive $X$ ray spectroscopy (WD-XRF) is an alternative analytical technique widely used for quantitative determination of the chemical composition in several type of geological or industrial samples: rocks, soils, sediments, cement (Dirken 2003). WD-XRF is a fast and reliable solution that covers a large part of the periodic table, in addition to having a field of application ranging from $\%$ up to ppm (Rousseau et al 1996).

Few studies based on the comparison of the XRF and ICP data are reported in literature (Amorosi e Sammartino 2011, Bettinelli et al. 2003, Chander et al. 2008) resulting in a complex picture. Indeed if Bettinelli reports a good agreement for sediments and less for rocks, either Amorosi and Sammartino and Chander clearly observe some problem for soil analysis. The ICP data indeed are strongly related to the digestion procedure, that can be often incomplete. As Amorosi and Sammartino emphasize, it's therefore important harmonizing the data from different analytical methods, a problem often overlooked.

The discrepancy between data from different analytical techniques results in significant consequences in the classification of environmental samples with respect to the legal limits. For this reason the argument is worthy of further information.

In the present work a comparison between the data obtained by XRF and ICP analysis on sediments and soils is reported.

\section{Materials and Methods}

All samples were grinded and homogenized in a mill with agate balls, before the ICP and XRF analysis. The particle 
Table 1. Quality parameter for the method of mineralization and DRC-ICP-MS analysis

\begin{tabular}{|c|c|c|c|c|c|c|c|}
\hline analyte & LOD & LOQ & precision & $\%$ recovery & $\%$ recovery spiked & $\begin{array}{c}\% \text { recovery trace } \\
\text { metals-Sandy } \\
\text { Clay 1 }\end{array}$ & $\begin{array}{c}\text { \% recovery } \\
\text { spiked soil } \\
\text { samples }\end{array}$ \\
\hline $\mathrm{ng} / \mathrm{g}$ & $\mathrm{ng} / \mathrm{g}$ & $\%$ & $\mathrm{TH} 2$ & sediment samples & 102 & $87 \pm 10$ \\
\hline $\mathrm{Cr}$ & 160 & 489 & 0.2 & 92 & $99 \pm 2$ & 100 & $102 \pm 30$ \\
\hline $\mathrm{Ni}$ & 73 & 209 & 1.9 & 107 & $107 \pm 3$ & 95 & $106 \pm 9$ \\
\hline $\mathrm{As}$ & 53 & 93 & 1.4 & 109 & $107 \pm 5$ & 103 & $85 \pm 20$ \\
\hline $\mathrm{Cd}$ & 56 & 74 & 11.3 & 107 & $108 \pm 4$ & 104 & $74 \pm 11$ \\
\hline
\end{tabular}

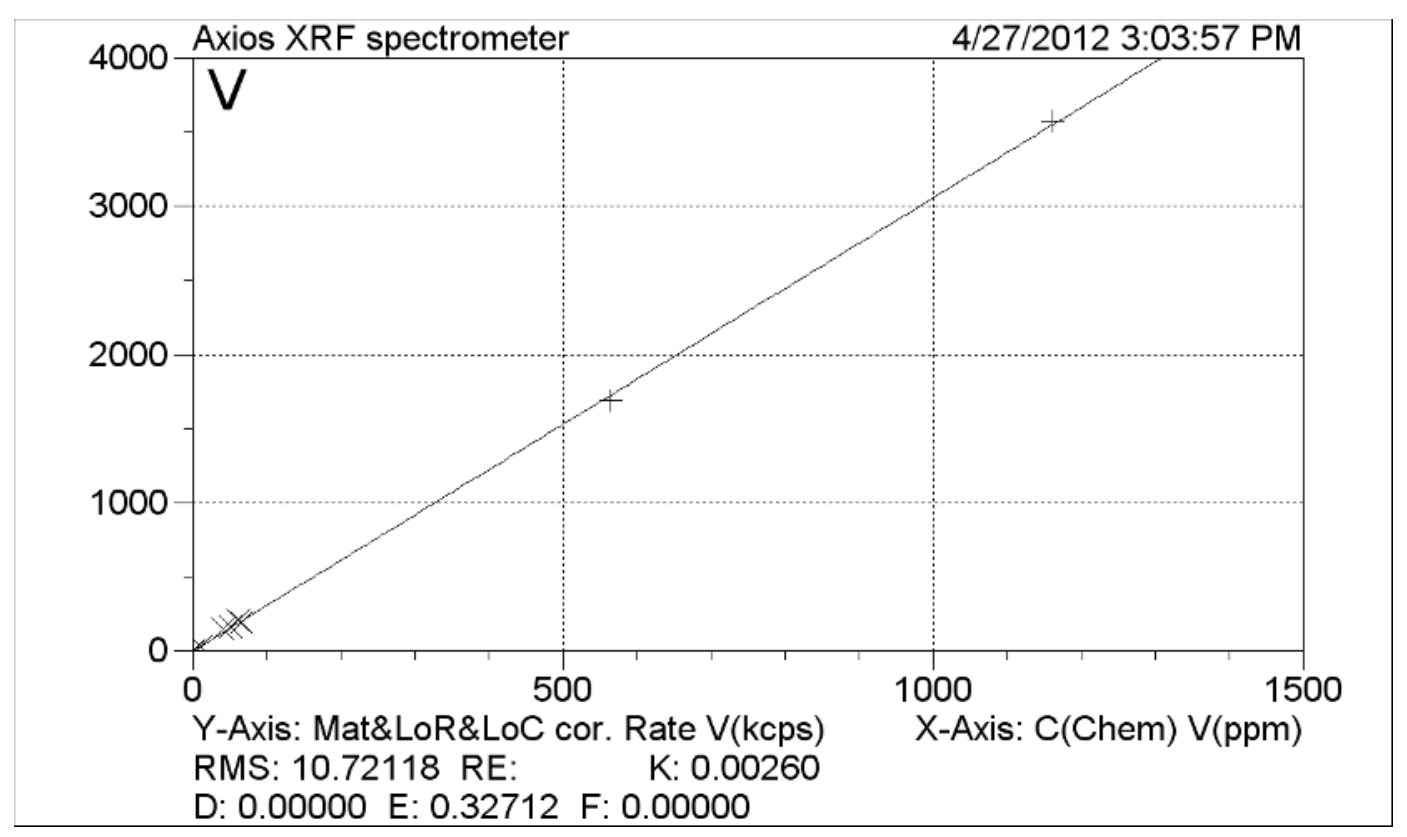

Fig. 1. XRF calibration line for Vanadium. The CRM data used for the validation are also displayed $(\times)$.

size distribution was monitored using the particle size analyzer Coulter LS1300.

Based on EPA 3052 we developed a method for sediments microwave mineralization at $200^{\circ} \mathrm{C}$, using $\mathrm{HNO}_{3}: \mathrm{HF}: \mathrm{H}_{2} \mathrm{O}_{2} \quad(16: 1: 2)$ in a $\mathrm{MW}$ digestion system Milestone Ethos One, using HPR-1000 vessels. All reagents were ultrapure grade for trace analysis and water was purified by a MilliQ system. We analyzed the digestate by means of DRC-ICP-MS (Perkin Elmer mod. Elan DRC-e). Instrumental condition were optimized for the best LODs that were reached by using a reaction gas: oxigen for detection of $\mathrm{As}\left({ }^{91} \mathrm{AsO}\right)$ and ${ }^{114} \mathrm{Cd}$, methane for ${ }^{60} \mathrm{Ni}$ and ${ }^{52} \mathrm{Cr}$. ${ }^{115}$ Indium was used as internal standard. The method was validated using two certified reference materials (CRM): TH2 (Toronto Harbour Sediment for Trace Elements - National Water Research Institute) and Trace metals-Sandy Clay1 (CRM049- RTC).

Pressed pellets samples were prepared for XRF analysis by mixing with wax (4:1) and pressing them using a manual press.

XRF analysis were made with a PANalytical Axios
Advanced spectrometer equipped with a $4 \mathrm{~kW}$ Rh anode X-ray tube, using the PANalytical Pro Trace package. This is a dedicated solution for the trace analysis of 43 elements based on the measurements collected on a set of high quality standards. Pro Trace performances were assessed by the analysis of several CRM (Govindaraju 1994).

\section{Results and Discussion}

We developed a mineralization method, for sediments, $\mathrm{HCl}$-free to avoid interferences determined by chlorine adducts in ICP-MS. The use of hydrofluoric acid in small quantities did not involve difficulties and its use was recommended in the literature for the effects generated on the recovery of different elements in several matrices (Baffi et al. 2002 for lichens, Sandroni and Smith 2002 for sludge, soil and sediment, Wang et al. 2004 and Xu et al 2005 for coal). The performance of the whole method of mineralization and DRC-ICP-MS analysis were assessed for precision, accuracy, limit of detection, 


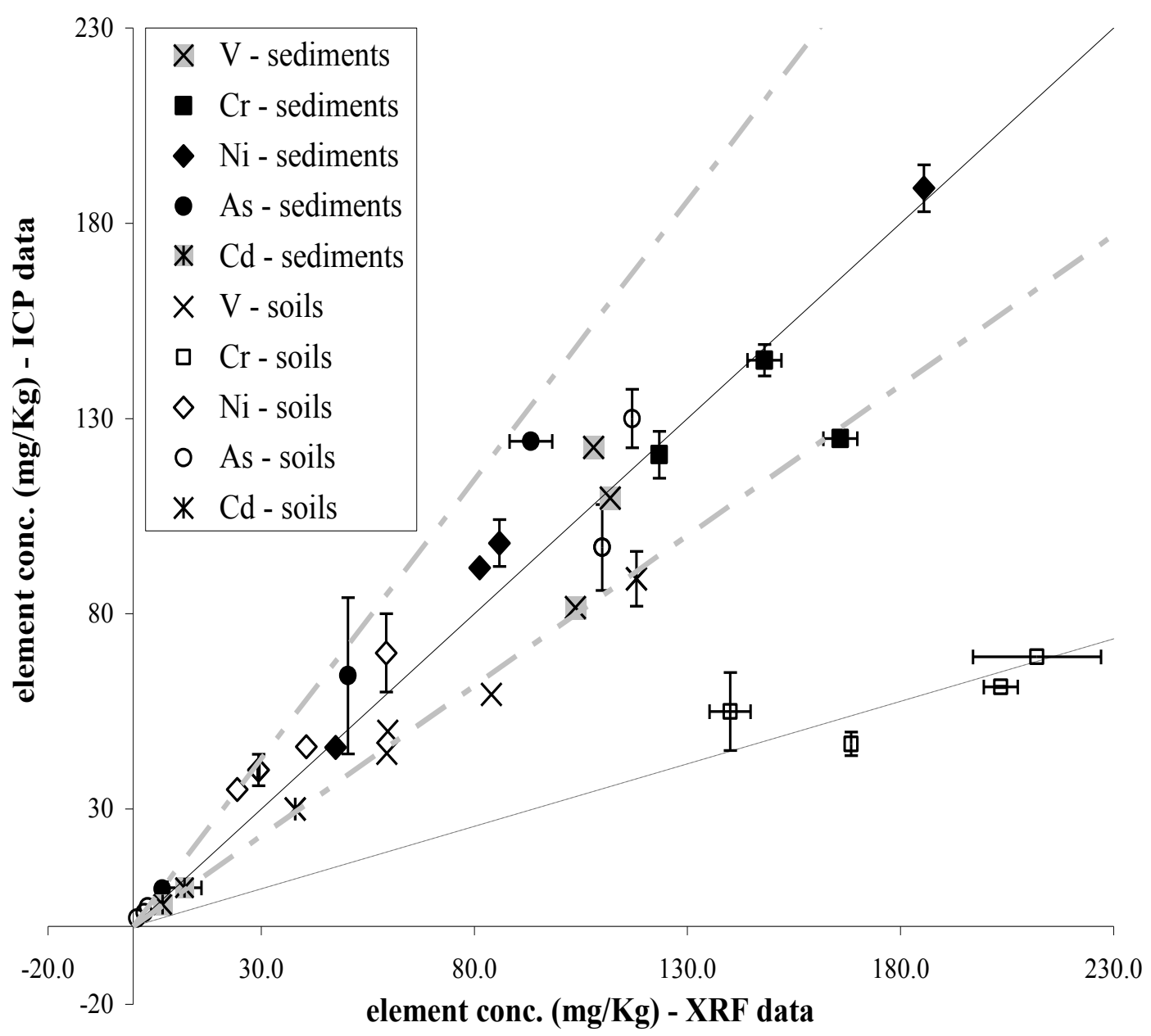

Fig. 2. Comparison between quantitative XRF and DRC-ICP-MS data for sediments and soils

(LOD) and limit of quantitation (LOQ). Precision was evaluated by comparing the results of multiple repetitions. Accuracy was expressed either as a percentage recovery of sediment spiked samples and as a percentage of the recovery of a certified sediment reference materials ("TH2"). LOD and LOQ were based on the results of many blank tests.

Similarly the method performances were valued when applied to soils, assessing accuracy by the analysis of spiked soil samples and certified soil reference materials ("Trace metals - Sandy Clay 1").

An overview of the resulting quality parameters is summarized in Table 1.

In the XRF Pro Trace calibration process particular attention has been paid to the determination of spectral line overlap corrections, background treatment and absorption effect corrections. The matrix correction was based on the use of the mass absorption coefficients (MAC), calculated following the Nesbitt method (Nesbitt et al 1976).

In Fig. 1 the calculated calibration line for vanadium, one of the elements under investigation, is reported. The results of CRM measurements $(\times)$ used for validation are also shown.

Both ICP and XRF analysis were applied to sediment and soil samples and to the CRM TH2.

Fig. 2 shows the results obtained by the two techniques. Each point represents the average of several repetitions (preparations and measures) and error bars their standard deviation. The straight line represents the hypothetical overlap of the results of the two techniques and the dashed-dotted gray lines represent a spread of $30 \%$ respect to "ideal" straight line.

Most of the reported data shows good agreement between ICP and XRF results and are within the range delimited by dashed-dotted gray lines.

On the other hand a strong deviation is observed for chromium on soil samples. The achieved results are aligned along the dashed line, representing an average of $32 \%$ of the XRF detectable content.

Incomplete chromium recovery by aqua regia digestion was observed by Amorosi and Sammartino for 
soil sample, while Bettinelli, using a mixture similar to the one we use, showed a complete chromium recovery for sediment samples, but did not report that obtained for rock samples. Chander suggested that differences in chromium extractable content from soils by different methods can be explained by differences in contamination source as well as sample $\mathrm{pH}$, organic carbon and clay content.

\section{Conclusion}

The comparison of data obtained by XRF and ICP analysis on soils and sediments, mineralized with a mixture of nitric acid, hydrofluoric acid and hydrogen peroxide shows good agreement for arsenic, cadmium, nickel and vanadium, indicating that this technique of extraction/mineralization allows the determination of the total content of these contaminants in the analyzed soil and sediment samples. For chromium the agreement is observed for sediment, while its recovery in soils is an average of $32 \%$, compared to the XRF detectable content.

This highlights the importance for a multi technique approach on trace analysis of environmental samples.

\section{Acknowledgements}

The authors thank Eugenio Casini (PANalytical s.r.1.) for his valuable support on XRF and Piernatale Casali (Eni S.p.A.) for CRM supply.

\section{References}

Amorosi A., Sammartino I. Assessing natural contents of hazardous metals in soils by different analytical methods and its impact on environmental legislative measures. Int. J. Environment and pollution 2011; 46: $164-177$

Baffi C., Bettinelli M., Beone GM., Spezia S. Comparison of different analytical procedures in the determination of trace elements in lichens. Chemosphere 2002; 48: 299-306

Bettinelli M., Beone G.M., Baffi C., Spezia S., Nassisi A. Characterization of environmental Samples in an Ophiolitic Area of Nortern Italy Using ICP-OES, ICP-MS, and XRF. Atomic Spectroscopy 2003; 24: $159-168$
Chander K, Hartmann G., Joergensen R.G., Khan K.S., Lamersdorf N. Comparison of methods for measuring heavy metals and total phosphorus in soil contaminated by different sources. Archives of Agronomy and Soil Science. 2008; 54:413-422

Dirken M.W. Determining Trace Concentration. Word Cement 2003; 34: 64-69

Govindaraju K. Compilation of working values and samples description for 383 geostandards. Geostandards Newsletters 1994; 18: 1-158

Nesbitt R.W., Mastins H., Stolz G.W., Bruce D.R. Matrix corrections in trace-element analysis by X-Ray Fluorescence: an extension of the Compton scattering technique to long wavelengths. Chemical Geology 1976; 18:203-213

Nouri M. Hassan, Pat E. Rasmussen, Ewa Dabek-Zlotorzynska, Valbona Celo, Heidi Chen. Analysis of Environmental Samples using Microwave-Assisted Acid Digestion and Inductively Coupled Plasma Mass Spectrometry: Maximizing Total Element Recoveries. Water Air Soil Pollut 2007; 178: 323-334

Quevauviller Ph., van der Sloot H.A., Ure A., Muntau H., Gomez A., Rauret G. Conclusion of the workshop: harmonization of leaching/extraction tests for environmental risk assessment. Science of The Total Environment 1996; 178: 133-139

Rousseau R. M., Willis J.P., Duncan A.R. Practical XRF Calibration Procedures for Major an Trace Elements. X Ray Spectrometry 1996; 25: 179-189

Sandroni V., Smith C.M. Microwave digestion of sludge, soil and sediment samples for metal analysis by inductively coupled plasma-atomic emission spectrometry. Analytica Chimica Acta 2002; 468: 335-344

Xu YH, Iwashita A., Nakajima T., Yamashita H.,; Takanashi H., Ohki A. Effect of HF addition on the microwave-assisted acid-digestion for the determination of metals in coal by inductively coupled plasma-atomic emission spectrometry. Talanta 2005; 66: 58-64

Wang J., Nakazato T., Kinya S., Yamada O., Tao H., Saito I. Microwave digestion with $\mathrm{HNO} 3 / \mathrm{H} 2 \mathrm{O} 2$ mixture at high temperatures for determination of trace elements in coal by ICP-OES and ICP-MS. Analytica Chimica Acta 2004; 514:115-124. 